\title{
A Sharper Estimate on the Betti Numbers of Sets Defined by Quadratic Inequalities
}

\author{
Saugata Basu • Michael Kettner
}

Received: 30 April 2006 / Revised: 27 April 2007 /

Published online: 19 September 2007

(C) Springer Science+Business Media, LLC 2007

Abstract In this paper we consider the problem of bounding the Betti numbers, $b_{i}(S)$, of a semi-algebraic set $S \subset \mathbb{R}^{k}$ defined by polynomial inequalities $P_{1} \geq 0$, $\ldots, P_{s} \geq 0$, where $P_{i} \in \mathbb{R}\left[X_{1}, \ldots, X_{k}\right], s<k$, and $\operatorname{deg}\left(P_{i}\right) \leq 2$, for $1 \leq i \leq s$. We prove that for $0 \leq i \leq k-1$,

$$
\begin{aligned}
b_{i}(S) & \leq \frac{1}{2}+(k-s)+\frac{1}{2} \cdot \sum_{j=0}^{\min \{s+1, k-i\}} 2^{j}\left(\begin{array}{c}
s+1 \\
j
\end{array}\right)\left(\begin{array}{c}
k \\
j-1
\end{array}\right) \\
& \leq \frac{3}{2} \cdot\left(\frac{6 e k}{s}\right)^{s}+k
\end{aligned}
$$

This improves the bound of $k^{O(s)}$ proved by Barvinok (in Math. Z. 225:231-244, 1997). This improvement is made possible by a new approach, whereby we first bound the Betti numbers of non-singular complete intersections of complex projective varieties defined by generic quadratic forms, and use this bound to obtain bounds in the real semi-algebraic case.

Keywords Betti numbers · Quadratic inequalities · Semi-algebraic sets

The first author was supported in part by an NSF grant CCF-0634907. The second author was partially supported by NSF grant CCF-0634907 and the European RTNetwork Real Algebraic and Analytic Geometry, Contract No. HPRN-CT-2001-00271.

S. Basu $(\bowtie) \cdot$ M. Kettner School of Mathematics, Georgia Institute of Technology, Atlanta, GA 30332, USA e-mail: saugata@math.gatech.edu

M. Kettner

e-mail: mkettner@math.gatech.edu 


\section{Introduction}

The topological complexity of semi-algebraic sets, measured by their Betti numbers (ranks of their singular homology groups), has been the subject of many investigations. For any topological space $X$, we will denote by $b_{i}(X)=b_{i}\left(X, \mathbb{Z}_{2}\right)$ the $i$-th Betti number of $X$ with $\mathbb{Z}_{2}$-coefficients, and we will denote by $b(X)$ the sum $\sum_{i \geq 0} b_{i}(X)$. Note that, since the homology groups of a semi-algebraic set $S \subset \mathbb{R}^{k}$ are finitely generated, it follows from the Universal Coefficients Theorem, that $b_{i}\left(S, \mathbb{Z}_{2}\right) \geq b_{i}(S, \mathbb{Z})$, where $b_{i}(S, \mathbb{Z})$ are the ordinary Betti numbers of $S$ with integer coefficients (see [15], Corollary 3.A6 (b)). Hence, the bounds proved in this paper also apply to the ordinary Betti numbers. However, our results do not yield any information on the Betti numbers of $S$ with $\mathbb{Z}_{p}$-coefficients, for $p \neq 2$. (The use of $\mathbb{Z}_{2}$-coefficients is necessitated by our use of Smith inequalities in the proof of the main theorem.)

The initial result on bounding the Betti numbers of semi-algebraic sets defined by polynomial inequalities was proved independently by Oleinik and Petrovskii [19], Thom [20] and Milnor [18]. They proved:

Theorem 1.1 [18-20] Let

$$
\mathcal{P}=\left\{P_{1}, \ldots, P_{s}\right\} \subset \mathbb{R}\left[X_{1}, \ldots, X_{k}\right]
$$

with $\operatorname{deg}\left(P_{i}\right) \leq d, 1 \leq i \leq s$ and let $S \subset \mathbb{R}^{k}$ be the set defined by

$$
P_{1} \geq 0, \ldots, P_{s} \geq 0 \text {. }
$$

Then,

$$
b(S)=O(s d)^{k}
$$

Notice that the above bound is exponential in $k$ and this exponential dependence is unavoidable (see Example 1.2 below). See also [5, 9, 12] for more recent work extending the above bound to more general classes of semi-algebraic sets.

\subsection{Semi-algebraic Sets Defined by Quadratic Inequalities}

In this paper we consider a restricted class of semi-algebraic sets-namely, semialgebraic sets defined by quadratic inequalities. Since sets defined by linear inequalities have no interesting topology, sets defined by quadratic inequalities can be considered to be the simplest class of semi-algebraic sets which can have non-trivial topology. Such sets are in fact quite general, as every semi-algebraic set can be defined by (quantified) formulas involving only quadratic polynomials (at the cost of increasing the number of variables and the size of the formula). Moreover, as in the case of general semi-algebraic sets, the Betti numbers of such sets can be exponentially large as can be seen in the following example.

Example 1.2 The set $S \subset \mathbb{R}^{k}$ defined by

$$
X_{1}\left(X_{1}-1\right) \geq 0, \ldots, X_{k}\left(X_{k}-1\right) \geq 0,
$$

has $b_{0}(S)=2^{k}$. 
However, it turns out that for a semi-algebraic set $S \subset \mathbb{R}^{k}$ defined by $s$ quadratic inequalities, it is possible to obtain upper bounds on the Betti numbers of $S$ which are polynomial in $k$ and exponential only in $s$. The first such result was proved by Barvinok who proved the following theorem.

Theorem 1.3 [2] Let $S \subset \mathbb{R}^{k}$ be defined by $P_{1} \geq 0, \ldots, P_{S} \geq 0, \operatorname{deg}\left(P_{i}\right) \leq 2,1 \leq$ $i \leq s$. Then, $b(S) \leq k^{O(s)}$.

Theorem 1.3 is proved using a duality argument that interchanges the roles of $k$ and $s$, and reduces the original problem to that of bounding the Betti numbers of a semi-algebraic set in $\mathbb{R}^{s}$ defined by $k^{O(1)}$ polynomials of degree at most $k$. One can then use Theorem 1.1 to obtain a bound of $k^{O(s)}$. The constant hidden in the exponent of the above bound is at least two. Also, the bound in Theorem 1.3 is polynomial in $k$ but exponential in $s$. The exponential dependence on $s$ is unavoidable as remarked in [2], but the implied constant (which is at least two) in the exponent of Barvinok's bound is not optimal.

Using Barvinok's result, as well as inequalities derived from the Mayer-Vietoris sequence, a polynomial bound (polynomial both in $k$ and $s$ ) was proved in [5] on the top few Betti numbers of a set defined by quadratic inequalities. More precisely the following theorem is proved there.

Theorem 1.4 [5] Let $\ell>0$ and $\mathrm{R}$ a real closed field. Let $S \subset \mathrm{R}^{k}$ be defined by

$$
P_{1} \geq 0, \ldots, P_{s} \geq 0
$$

with $\operatorname{deg}\left(P_{i}\right) \leq 2$. Then,

$$
b_{k-\ell}(S) \leq\left(\begin{array}{l}
s \\
\ell
\end{array}\right) k^{O(\ell)}
$$

Notice that for fixed $\ell$, the bound in Theorem 1.4 is polynomial in both $s$ as well as $k$.

Apart from their intrinsic mathematical interest (in distinguishing the semialgebraic sets defined by quadratic inequalities from general semi-algebraic sets), the bounds in Theorems 1.3 and 1.4 have motivated recent work on designing polynomial time algorithms for computing topological invariants of semi-algebraic sets defined by quadratic inequalities (see $[3,6,7,10,13]$ ). Traditionally an important goal in algorithmic semi-algebraic geometry has been to design algorithms for computing topological invariants of semi-algebraic sets, whose worst-case complexity matches the best upper bounds known for the quantity being computed. It is thus of interest to tighten the bounds on the Betti numbers of semi-algebraic sets defined by quadratic inequalities, as has been done recently in the case of general semi-algebraic sets (see for example $[5,9,12])$.

\subsection{Brief Outline of Our Method}

In this paper we use a new method to bound the Betti numbers of semi-algebraic sets defined by quadratic inequalities. Our method is to first bound the Betti numbers of complex projective varieties which are non-singular complete intersections 
defined by quadratic forms in general position. It is a well known fact from complex geometry, (see for instance, [16], p. 122) that the Betti numbers of a complex projective variety which is a non-singular complete intersection depend only on the sequence of degrees and the number of variables of the polynomials defining the variety. Moreover, there exist precise formulas for the Betti numbers of such varieties, using well-known techniques from algebraic geometry (see Theorem 2.7 below).

Our strategy for bounding the Betti numbers of semi-algebraic sets in $\mathbb{R}^{k}$ defined by $s$ quadratic inequalities is as follows. Using certain infinitesimal deformations we first reduce the problem to bounding the Betti numbers of another closed and bounded semi-algebraic set defined by a new family quadratic polynomials. We then use inequalities obtained from the Mayer-Vietoris exact sequence to further reduce the problem of bounding the Betti numbers of this new semi-algebraic set, to the problem of bounding the Betti numbers of the real projective varieties defined by each $\ell$-tuple, $\ell \leq s$, of the new polynomials. The new family of polynomials also has the property that the complex projective variety defined by each $\ell$-tuple, $\ell \leq k$, of these polynomials is a non-singular complete intersection. As mentioned above we have precise information about the Betti numbers of these complex complete intersections. An application of Smith inequalities then allows us to obtain bounds on the Betti numbers of the real parts of these varieties and as a result on the Betti numbers of the original semi-algebraic set. Because of the direct nature of our proof we are able to remove the constant in the exponent in the bounds proved in $[2,5]$ and this constitutes the main contribution of this paper.

Remark 1.5 We remark here that the technique used in this paper was proposed as a possible alternative method by Barvinok in [2], who did not pursue this further in that paper. Also, Benedetti, Loeser, and Risler [11] used a similar technique for proving upper bounds on the number of connected components of real algebraic sets in $\mathbb{R}^{k}$ defined by polynomials of degrees bounded by $d$. However, these bounds (unlike the situation considered in this paper) are exponential in $k$. Finally, there exists another possible method for bounding the Betti numbers of semi-algebraic sets defined by quadratic inequalities, using a spectral sequence argument due to Agrachev [1]. However, this method also produces a non-optimal bound of the form $k^{O(s)}$ (similar to Barvinok's bound) where the constant in the exponent is at least two. We omit the details of this argument referring the reader to [6] for an indication of the proof (where the case of computing, and as a result, bounding the Euler-Poincaré characteristics of such sets is worked out in full details).

We prove the following theorem.

Theorem 1.6 Let $\mathcal{P}=\left\{P_{1}, \ldots, P_{s}\right\} \subset \mathbb{R}\left[X_{1}, \ldots, X_{k}\right], s<k$. Let $S \subset \mathbb{R}^{k}$ be defined by

$$
P_{1} \geq 0, \ldots, P_{s} \geq 0
$$

with $\operatorname{deg}\left(P_{i}\right) \leq 2$. Then, for $0 \leq i \leq k-1$,

$$
\frac{1}{2}+(k-s)+\frac{1}{2} \cdot \sum_{j=0}^{\min \{s+1, k-i\}} 2^{j}\left(\begin{array}{c}
s+1 \\
j
\end{array}\right)\left(\begin{array}{c}
k \\
j-1
\end{array}\right) \leq \frac{3}{2} \cdot\left(\frac{6 e k}{s}\right)^{s}+k
$$


As a consequence of Theorem 1.6 we get a new bound on the sum of the Betti numbers, which we state for the sake of completeness.

Corollary 1.7 Let $\mathcal{P}=\left\{P_{1}, \ldots, P_{s}\right\} \subset \mathbb{R}\left[X_{1}, \ldots, X_{k}\right], s<k$. Let $S \subset \mathbb{R}^{k}$ be defined by

$$
P_{1} \geq 0, \ldots, P_{s} \geq 0
$$

with $\operatorname{deg}\left(P_{i}\right) \leq 2$. Then,

$$
b(S) \leq k\left(\frac{1}{2}+(k-s)+\frac{1}{2} \cdot \sum_{j=0}^{\min \{s+1, k-i\}} 2^{j}\left(\begin{array}{c}
s+1 \\
j
\end{array}\right)\left(\begin{array}{c}
k \\
j-1
\end{array}\right)\right) .
$$

The rest of the paper is organized as follows. In Sect. 2, we recall some well known results from complex algebraic geometry on the Betti numbers of non-singular complex projective varieties which are complete intersections, and also some classical results from algebraic topology which we need for the proof of our main result. In Sect. 3, we prove Theorem 1.6. Finally, in Sect. 4 we state some open problems.

\section{Mathematical Preliminaries}

In this section we recall a few basic facts about Betti numbers and complex projective varieties which are non-singular complete intersections, as well as fix some notations.

Throughout the paper, $\mathbb{P}_{\mathbb{R}}^{k}$ (respectively, $\mathbb{P}_{\mathbb{C}}^{k}$ ) denotes the real (respectively, complex) projective space of dimension $k, \mathbf{S}^{k}$ (resp., $\mathbf{B}^{k+1}$ ) denotes the sphere (resp., closed ball) centered at the origin of unit radius in $\mathbb{R}^{k+1}$. For any polynomial $P \in \mathbb{R}\left[X_{1}, \ldots, X_{k}\right]$, let be $P^{h}\left(X_{1}, \ldots, X_{k+1}\right)=X_{k+1}^{d} P\left(\frac{X_{1}}{X_{k+1}}, \ldots, \frac{X_{k}}{X_{k+1}}\right)$, where $d$ is the total degree of $P$, the homogenization of $P$ with respect to $X_{k+1}$.

For any family of polynomials $\mathcal{P}=\left\{P_{1}, \ldots, P_{\ell}\right\} \subset \mathbb{R}\left[X_{1}, \ldots, X_{k}\right]$, and $Z \subset \mathbb{R}^{k}$, we denote by $\operatorname{Zer}(\mathcal{P}, Z)$ the set of common zeros of $\mathcal{P}$ in $Z$. Moreover, for any family of homogeneous polynomials $\mathcal{Q}=\left\{Q_{1}, \ldots, Q_{\ell}\right\} \subset \mathbb{R}\left[X_{1}, \ldots, X_{k+1}\right]$, we denote by $\operatorname{Zer}\left(\mathcal{Q}, \mathbb{P}_{\mathbb{R}}^{k}\right)\left(\right.$ resp., $\left.\operatorname{Zer}\left(\mathcal{Q}, \mathbb{P}_{\mathbb{C}}^{k}\right)\right)$ the set of common zeros of $\mathcal{Q}$ in $\mathbb{P}_{\mathbb{R}}^{k}\left(\right.$ resp., $\left.\mathbb{P}_{\mathbb{C}}^{k}\right)$.

2.1 Some Results from Algebraic Topology

Let

$$
\mathcal{Q}=\left\{Q_{1}, \ldots, Q_{\ell}\right\} \subset \mathbb{R}\left[X_{1}, \ldots, X_{k+1}\right]
$$

be a set of homogeneous polynomials. Denote by $\mathcal{Q}_{J}$ the set $\left\{Q_{j} \mid j \in J\right\}$ for $J \subset$ $\{1, \ldots, \ell\}$. We have the following inequality which is a consequence of the MayerVietoris exact sequence.

Proposition 2.1 Let $Z \subset \mathbb{R}^{k+1}$. For $0 \leq i \leq k-1$,

$$
b_{i}\left(\bigcup_{j=1}^{\ell} \operatorname{Zer}\left(Q_{j}, Z\right)\right) \leq \sum_{j=1}^{i+1} \sum_{J \subset\{1, \ldots, \ell\},|J|=j} b_{i-j+1}\left(\operatorname{Zer}\left(\mathcal{Q}_{J}, Z\right)\right) .
$$


Proof See [5], Lemma 2.

We also use the well-known Alexander duality theorem which relates the Betti numbers of a compact subset of a sphere to those of its complement.

Theorem 2.2 (Alexander Duality) Let $r>0$. For any closed subset $A \subset \mathbf{S}^{k}$,

$$
\mathrm{H}_{i}\left(\mathbf{S}^{k} \backslash A\right) \approx \tilde{\mathrm{H}}^{k-i-1}(A),
$$

where $\tilde{\mathrm{H}}^{i}(A), 0 \leq i \leq k-1$, denotes the reduced cohomology group of $A$.

Proof See [17], Theorem 6.6.

The following proposition relates the Betti numbers of a finite CW-complex to its double cover. Note that the proposition is no longer true for Betti numbers with $\mathbb{Z}$-coefficients. A simple counterexample is provided by the 2-torus which is a double cover of the Klein bottle, for which the stated inequality is not true for $i=2$ for Betti numbers with $\mathbb{Z}$-coefficients.

Proposition 2.3 Let $X$ be a finite simplicial complex and $f: \tilde{X} \rightarrow X$ a double cover of $X$. Then, for each $i \geq 0$,

$$
b_{i}(\tilde{X}) \leq 2 b_{i}(X) .
$$

Proof Let $\mathrm{C}_{\bullet}\left(X, \mathbb{Z}_{2}\right)$ and $\mathrm{C}_{\bullet}\left(\tilde{X}, \mathbb{Z}_{2}\right)$ denote the simplicial chain complex of $X$ and $\tilde{X}$ respectively with coefficients in $\mathbb{Z}_{2}$. Let

$$
\phi_{\bullet}: \mathrm{C}_{\bullet}\left(X, \mathbb{Z}_{2}\right) \longrightarrow \mathrm{C}_{\bullet}\left(\tilde{X}, \mathbb{Z}_{2}\right)
$$

denote the chain map sending each simplex of $X$ to the sum of its two preimages in $\tilde{X}$. Let

$$
\psi_{\bullet}: \mathrm{C}_{\bullet}\left(\tilde{X}, \mathbb{Z}_{2}\right) \longrightarrow \mathrm{C}_{\bullet}\left(X, \mathbb{Z}_{2}\right)
$$

be the chain map induced by the covering map $f$.

It is an easy exercise to check that the following sequence is exact,

$$
0 \longrightarrow \mathrm{C}_{\bullet}\left(X, \mathbb{Z}_{2}\right) \stackrel{\phi_{\bullet}}{\longrightarrow} \mathrm{C}_{\bullet}\left(\tilde{X}, \mathbb{Z}_{2}\right) \stackrel{\psi_{\bullet}}{\longrightarrow} \mathrm{C}_{\bullet}\left(X, \mathbb{Z}_{2}\right) \longrightarrow 0 .
$$

The corresponding long exact sequence in homology,

$$
\cdots \longrightarrow \mathrm{H}_{i}\left(X, \mathbb{Z}_{2}\right) \longrightarrow \mathrm{H}_{i}\left(\tilde{X}, \mathbb{Z}_{2}\right) \longrightarrow \mathrm{H}_{i}\left(X, \mathbb{Z}_{2}\right) \longrightarrow \cdots
$$

gives the required inequality.

Finally, we state a version of the Smith inequality which plays a crucial role in the proof of the main theorem. Recall that for any compact topological space equipped with an involution, inequalities derived from the Smith exact sequences allows one to bound the sum of the Betti numbers (with $\mathbb{Z}_{2}$ coefficients) of the fixed point set of the 
involution by the sum of the Betti numbers (again with $\mathbb{Z}_{2}$ coefficients) of the space itself (see for instance, [21], p. 131). In particular, we have for a complex projective variety defined by real forms, with the involution taken to be complex conjugation, the following theorem.

Theorem 2.4 (Smith inequality) Let $\mathcal{Q} \subset \mathbb{R}\left[X_{1}, \ldots, X_{k+1}\right]$ be a family of homogeneous polynomials. Then,

$$
b\left(\operatorname{Zer}\left(\mathcal{Q}, \mathbb{P}_{\mathbb{R}}^{k}\right)\right) \leq b\left(\operatorname{Zer}\left(\mathcal{Q}, \mathbb{P}_{\mathbb{C}}^{k}\right)\right)
$$

\subsection{Complete Intersection Varieties}

Definition 2.5 A projective variety $X \subset \mathbb{P}_{\mathbb{C}}^{k}$ of codimension $n$ is a non-singular complete intersection if it is the intersection of $n$ non-singular hypersurfaces in $\mathbb{P}_{\mathbb{C}}^{k}$ that meet transversally at each point of the intersection.

Fix a $j$-tuple of natural numbers $\bar{d}=\left(d_{1}, \ldots, d_{j}\right)$. Let $X_{\mathbb{C}}=\operatorname{Zer}\left(\left\{Q_{1}, \ldots, Q_{j}\right\}\right.$, $\left.\mathbb{P}_{\mathbb{C}}^{k}\right)$, such that the degree of $Q_{i}$ is $d_{i}$, denote a complex projective variety of codimension $j$ which is a non-singular complete intersection.

Let $b(j, k, \bar{d})$ denote the sum of the Betti numbers with $\mathbb{Z}_{2}$ coefficients of $X_{\mathbb{C}}$. This is well defined since the Betti numbers only depend only on the degree sequence and not on the specific $X_{\mathbb{C}}$.

The function $b(j, k, \bar{d})$ satisfies the following (see [11]):

$$
b(j, k, \bar{d})= \begin{cases}c(j, k, \bar{d}) & \text { if } k-j \text { is even } \\ 2(k-j+1)-c(j, k, \bar{d}) & \text { if } k-j \text { is odd }\end{cases}
$$

where

$$
\begin{aligned}
& c(j, k, \bar{d}) \\
& \quad= \begin{cases}k+1 & \text { if } j=0, \\
d_{1} \ldots d_{j} & \text { if } j=k, \\
d_{k} c\left(j-1, k-1,\left(d_{1}, \ldots, d_{k-1}\right)\right)-\left(d_{k}-1\right) c(j, k-1, \bar{d}) & \text { if } 0<j<k .\end{cases}
\end{aligned}
$$

In the special case when each $d_{i}=2$, we denote by $b(j, k)=b(j, k,(2, \ldots, 2))$. We then have the following recurrence for $b(j, k)$.

$$
b(j, k)= \begin{cases}q(j, k) & \text { if } k-j \text { is even, } \\ 2(k-j+1)-q(j, k) & \text { if } k-j \text { is odd }\end{cases}
$$

where

$$
q(j, k)= \begin{cases}k+1 & \text { if } j=0, \\ 2^{j} & \text { if } j=k, \\ 2 q(j-1, k-1)-q(j, k-1) & \text { if } 0<j<k .\end{cases}
$$

Next, we show some properties of $q(j, k)$. 


\section{Lemma 2.6}

(1) $q(1, k)=k+1 / 2\left(1-(-1)^{k}\right)$ and $q(2, k)=(-1)^{k} k+k$.

(2) For $2 \leq j \leq k,|q(j, k)| \leq 2^{j-1}\left(\begin{array}{c}k \\ j-1\end{array}\right)$.

(3) For $2 \leq j \leq k$ and $k-j$ odd, $2(k-j+1)-q(j, k) \leq 2^{j-1}\left(\begin{array}{c}k \\ j-1\end{array}\right)$.

Proof The first part is shown by two easy computations and note that $2(k-2+1)-$ $q(2, k)=2 k-2$ if $k-2$ is odd. Hence, we can assume that the statements are true for $k-1$ and that $3 \leq j<k$. Note that for the special case $j=k-1$, we have that $2^{k-1} \leq 2^{k-2}\left(\begin{array}{l}k-1 \\ k-2\end{array}\right)$ since $k>2$.Then,

$$
\begin{aligned}
|q(j, k)| & =|2 q(j-1, k-1)-q(j, k-1)| \\
& \leq 2|q(j-1, k-1)|+|q(j, k-1)| \\
& \leq 2 \cdot 2^{j-2}\left(\begin{array}{c}
k-1 \\
j-2
\end{array}\right)+2^{j-1}\left(\begin{array}{c}
k-1 \\
j-1
\end{array}\right) \\
& =2^{j-1}\left(\begin{array}{c}
k \\
j-1
\end{array}\right)
\end{aligned}
$$

and, for $k-j$ odd,

$$
\begin{aligned}
2(k-j+1)-q(j, k)= & 2(k-j+1)-2 q(j-1, k-1)+q(j, k-1) \\
\leq & |2((k-1)-(j-1)+1)-q(j-1, k-1)| \\
& +|q(j-1, k-1)|+|q(j, k-1)| \\
\leq & 2^{j-2}\left(\begin{array}{c}
k-1 \\
j-2
\end{array}\right)+2^{j-2}\left(\begin{array}{c}
k-1 \\
j-2
\end{array}\right)+2^{j-1}\left(\begin{array}{c}
k-1 \\
j-1
\end{array}\right) \\
\leq & 2^{j-1}\left(\left(\begin{array}{c}
k-1 \\
j-2
\end{array}\right)+\left(\begin{array}{c}
k-1 \\
j-1
\end{array}\right)\right)=2^{j-1}\left(\begin{array}{c}
k \\
j-1
\end{array}\right) .
\end{aligned}
$$

Hence, we get the following bound for $b(j, k)$.

\section{Theorem 2.7}

(1) $b(1, k)= \begin{cases}q(0, k-1) & \text { if } k \text { is even, } \\ q(0, k) & \text { if } k \text { is odd. }\end{cases}$

(2) $b(j, k) \leq 2^{j-1}\left(\begin{array}{c}k \\ j-1\end{array}\right)$, for $2 \leq j \leq k$.

Proof Follows from Lemma 2.6.

Proposition 2.8 There exist a family $\mathcal{H}=\left\{H_{1}, \ldots, H_{s}\right\} \subset \mathbb{R}\left[X_{1}, \ldots, X_{k+1}\right], s<k$, of positive definite quadratic forms such that $\operatorname{Zer}\left(\mathcal{H}_{J}, \mathbb{P}_{\mathbb{C}}^{k}\right)$ is a non-singular complete intersection for every $J \subset\{1, \ldots, s\}$.

Proof Recall that the set of positive definite quadratic forms is open in the set of quadratic forms (over $\mathbb{R}$ ). Thus, we can choose a family $\mathcal{H}=\left\{H_{1}, \ldots, H_{s}\right\} \subset$ 
$\mathbb{R}\left[X_{1}, \ldots, X_{k+1}\right], s<k$, of positive definite quadratic forms such that their coefficients are algebraically independent over $\mathbb{Q}$. It follows by Bertini's Theorem (see [14], Theorem 17.16) that $\operatorname{Zer}\left(\mathcal{H}_{J}, \mathbb{P}_{\mathbb{C}}^{k+1}\right), J \subset\{1, \ldots, s\}$, is a non-singular complete intersection.

The following proposition allows to replace a set of real quadratic forms by another family obtained by infinitesimal perturbations of the original family and whose zero sets are non-singular complete intersections in complex projective space.

Proposition 2.9 Let

$$
\mathcal{Q}=\left\{Q_{1}, \ldots, Q_{s}\right\} \subset \mathbb{R}\left[X_{1}, \ldots, X_{k+1}\right],
$$

$s<k$, be a set of quadratic forms and let

$$
\mathcal{H}=\left\{H_{1}, \ldots, H_{s}\right\} \subset \mathbb{R}\left[X_{1}, \ldots, X_{k+1}\right]
$$

be a family of positive definite quadratic forms such that $\operatorname{Zer}\left(\mathcal{H}, \mathbb{P}_{\mathbb{C}}^{k}\right)$ is a non-singular complete intersection for every $J \subset\{1, \ldots, s\}$.

For $t \in \mathbb{C}$, let

$$
\begin{gathered}
\tilde{\mathcal{Q}}_{t}=\left\{\tilde{Q}_{t, 1}, \ldots, \tilde{Q}_{t, s}\right\} \quad \text { with } \\
\tilde{Q}_{t, i}=(1-t) Q_{i}+t H_{i} .
\end{gathered}
$$

Then, for all sufficiently small $\delta>0$, and any $J \subset\{1, \ldots, s\}$,

$$
\operatorname{Zer}\left(\tilde{\mathcal{Q}}_{\delta, J}, \mathbb{P}_{\mathbb{C}}^{k}\right)
$$

is a non-singular complete intersection.

Proof Let $J \subset\{1, \ldots, s\}$, and let $T_{J} \subset \mathbb{C}$ be defined by

$$
T_{J}=\left\{t \in \mathbb{C} \mid \operatorname{Zer}\left(\tilde{\mathcal{Q}}_{t, J}, \mathbb{P}_{\mathbb{C}}^{k}\right) \text { is a non-singular complete intersection }\right\} .
$$

Clearly, $T_{J}$ contains 1 . Moreover, since being a non-singular complete intersection is stable condition, $T_{J}$ must contain an open neighborhood of 1 in $\mathbb{C}$ and so must $T=\bigcap_{J \subset\{1, \ldots, s\}} T_{J}$. Finally, the set $T$ is constructible, since it can be defined by a first order formula. Since a constructible subset of $\mathbb{C}$ is either finite or the complement of a finite set (see for instance, [9], Corollary 1.25), $T$ must contain an interval $\left(0, t_{0}\right)$, $t_{0}>0$.

\section{Proof of Theorem 1.6}

In order to prove Theorem 1.6 we need what follows next:

Let $\mathcal{P}=\left\{P_{1}, \ldots, P_{s}\right\} \subset \mathbb{R}\left[X_{1}, \ldots, X_{k}\right], s<k$, with $\operatorname{deg}\left(P_{i}\right) \leq 2,1 \leq i \leq s$. Let $S \subset \mathbb{R}^{k}$ be the basic semi-algebraic set defined by $P_{1} \geq 0, \ldots, P_{s} \geq 0$. Let $P_{s+1}=(1 / \varepsilon)^{2}-\sum_{i=1}^{k} X_{i}^{2}$ and let $S_{\varepsilon} \subset \mathbb{R}^{k}$ be the basic semi-algebraic set defined by $P_{1} \geq 0, \ldots, P_{s} \geq 0, P_{s+1} \geq 0$.

Springer 
Proposition 3.1 For all sufficiently small $\varepsilon>0$, the homology groups of $S$ and $S_{\varepsilon}$ are isomorphic. Moreover, $S_{\varepsilon}$ is bounded.

Proof The proof follows from Hardt's triviality theorem (see [8], Theorem 5.45.) and is similar to the proof of Lemma 1 in [4].

Let $S_{\varepsilon}^{h}$ be the basic semi-algebraic set defined by $P_{1}^{h} \geq 0, \ldots, P_{s}^{h} \geq 0, P_{s+1}^{h} \geq 0$ in $\mathbf{S}^{k}$.

Lemma 3.2 For sufficiently small $\varepsilon>0$ and $0 \leq i \leq k$, we have

$$
b_{i}\left(S_{\varepsilon}\right)=\frac{1}{2} b_{i}\left(S_{\varepsilon}^{h}\right)
$$

Proof Note that $S_{\varepsilon}$ is bounded by Proposition 3.1 and $S_{\varepsilon}^{h}$ is the projection from the origin of the set $S_{\varepsilon} \times\{1\} \subset \mathbb{R}^{k} \times\{1\}$ onto the unit sphere in $\mathbb{R}^{k+1}$. Since $S_{\varepsilon}$ is bounded, the projection does not intersect the equator and consists of two disjoint copies (each homeomorphic to the set $S_{\varepsilon}$ ) in the upper and lower hemispheres.

We now fix a sufficiently small $\varepsilon>0$ and a family of polynomials that will be useful in what follows. By Proposition 2.8 we can choose a family $\mathcal{H}=\left\{H_{1}, \ldots, H_{s+1}\right\} \subset \mathbb{R}\left[X_{1}, \ldots, X_{k+1}\right]$ of positive definite quadratic forms such that $\operatorname{Zer}\left(\mathcal{H}_{J}, \mathbb{P}_{\mathbb{C}}^{k}\right)$ is a non-singular complete intersection for every $J \subset\{1, \ldots, s+1\}$.

Let $\delta>0$ and let $\tilde{P}_{i}=(1-\delta) P_{i}^{h}+\delta H_{i}, 1 \leq i \leq s+1$. Let $T_{\varepsilon, \delta}$ (resp., $\bar{T}_{\varepsilon, \delta}$ ) be the basic semi-algebraic set defined by $\tilde{P}_{1}>0, \ldots, \tilde{P}_{s}>0, \tilde{P}_{s+1}>0$ (resp., $\left.\tilde{P}_{1} \geq 0, \ldots, \tilde{P}_{S} \geq 0, \tilde{P}_{s+1} \geq 0\right)$ contained in the sphere $\mathbf{S}^{k}$.

Also, let

$$
\tilde{\mathcal{P}}=\left\{\tilde{P}_{1}, \ldots, \tilde{P}_{s}, \tilde{P}_{s+1}\right\}
$$

In the following, the notation $0<\delta \ll \varepsilon$ denotes "for all sufficiently small $\varepsilon>0$, and having chosen $\varepsilon$ for all sufficiently small $\delta>0$ ".

Lemma 3.3 For all sufficiently small $0<\delta \ll \varepsilon$ we have,

(1) the homology groups of $S_{\varepsilon}^{h}$ and $\bar{T}_{\varepsilon, \delta}$ are isomorphic,

(2) the homology groups of $T_{\varepsilon, \delta}$ and $\bar{T}_{\varepsilon, \delta}$ are isomorphic,

(3) for all $J \subset\{1, \ldots, s+1\}, \operatorname{Zer}\left(\tilde{\mathcal{P}}_{J}, \mathbb{P}_{\mathbb{C}}^{k}\right)$ is a non-singular complete intersection, and

(4) for all $J \subset\{1, \ldots, s+1\}, b_{i}\left(\operatorname{Zer}\left(\tilde{\mathcal{P}}_{J}, \mathbf{S}^{k}\right)\right) \leq 2 b_{i}\left(\operatorname{Zer}\left(\tilde{\mathcal{P}}_{J}, \mathbb{P}_{\mathbb{R}}^{k}\right)\right)$.

Proof For the first part note that $S_{\varepsilon}$ and $\bar{T}_{\varepsilon, \delta}$ have the same homotopy type using Lemma 16.17 in [8].

The second part is clear since by choosing any slightly smaller $0<\delta^{\prime}<\delta$, we have a retraction from $T_{\varepsilon, \delta}$ to $\bar{T}_{\varepsilon, \delta^{\prime}}$.

The third part follows from Proposition 2.9. 
For the last part, let $\pi: \mathbf{S}^{k} \rightarrow \mathbb{P}_{\mathbb{R}}^{k}$ be the double cover obtained by identifying antipodal points. Then the restriction of $\pi$ to $\operatorname{Zer}\left(\tilde{\mathcal{P}}_{J}, \mathbf{S}^{k}\right)$ gives a double cover,

$$
\pi: \operatorname{Zer}\left(\tilde{\mathcal{P}}_{J}, \mathbf{S}^{k}\right) \rightarrow \operatorname{Zer}\left(\tilde{P}_{J}, \mathbb{P}_{\mathbb{R}}^{k}\right)
$$

Now apply Proposition 2.3.

Proposition 3.4 For all sufficiently small $0<\delta \ll \varepsilon$ and for $0 \leq i \leq k-1$, we have

$$
b_{i}\left(T_{\varepsilon, \delta}\right) \leq 1+2(k-s)+\sum_{j=0}^{\min \{s+1, k-i\}} 2^{j}\left(\begin{array}{c}
s+1 \\
j
\end{array}\right)\left(\begin{array}{c}
k \\
j-1
\end{array}\right) .
$$

Proof First note that by Lemma 3.3 (3) $\operatorname{Zer}\left(\tilde{\mathcal{P}}_{J}, \mathbb{P}_{\mathbb{C}}^{k}\right)$ is a complete intersection for all $J \subset\{1, \ldots, s+1\}$.

For $0 \leq i \leq k-1$,

$$
b_{i}\left(T_{\varepsilon, \delta}\right) \leq b_{i}\left(\mathbf{S}^{k} \backslash \bigcup_{i=1}^{s+1} \operatorname{Zer}\left(\tilde{P}_{i}, \mathbf{S}^{k}\right)\right) \leq 1+b_{k-1-i}\left(\bigcup_{i=1}^{s+1} \operatorname{Zer}\left(\tilde{P}_{i}, \mathbf{S}^{k}\right)\right)
$$

where the first inequality is a consequence of the fact that, $T_{\varepsilon, \delta}$ is an open subset of $\mathbf{S}^{k} \backslash \bigcup_{i=1}^{s+1} \operatorname{Zer}\left(\tilde{P}_{i}, \mathbf{S}^{k}\right)$ and disconnected from its complement in $\mathbf{S}^{k} \backslash$ $\bigcup_{i=1}^{s+1} \operatorname{Zer}\left(\tilde{P}_{i}, \mathbf{S}^{k}\right)$, and the last inequality follows from Theorem 2.2 (Alexander Duality). It follows from Proposition 2.1, Lemma 3.3 (4) and Theorem 2.4 (Smith inequality), that

$$
\begin{aligned}
b_{i}\left(T_{\varepsilon, \delta}\right) & \leq 1+\sum_{j=1}^{k-i} \sum_{|J|=j} b_{k-i-j}\left(\operatorname{Zer}\left(\tilde{\mathcal{P}}_{J}, \mathbf{S}^{k}\right)\right) \\
& \leq 1+2 \cdot \sum_{j=1}^{k-i} \sum_{|J|=j} b_{k-i-j}\left(\operatorname{Zer}\left(\tilde{\mathcal{P}}_{J}, \mathbb{P}_{\mathbb{R}}^{k}\right)\right) \\
& \leq 1+2 \cdot \sum_{j=1}^{\min \{s+1, k-i\}} \sum_{|J|=j} b\left(\operatorname{Zer}\left(\tilde{\mathcal{P}}_{J}, \mathbb{P}_{\mathbb{C}}^{k}\right)\right) .
\end{aligned}
$$

Note that for $j \leq s+1$ the number of possible $j$-ary intersections is equal to $\left(\begin{array}{c}s+1 \\ j\end{array}\right)$ and using Theorem 2.7, we conclude

$$
\begin{aligned}
b_{i}\left(T_{\varepsilon, \delta}\right) & \leq 1+2 \cdot \sum_{j=1}^{\min \{s+1, k-i\}}\left(\begin{array}{c}
s+1 \\
j
\end{array}\right) b(j, k) \\
& \leq 1+2(k+1)+2 \cdot \sum_{j=2}^{\min \{s+1, k-i\}}\left(\begin{array}{c}
s+1 \\
j
\end{array}\right) 2^{j-1}\left(\begin{array}{c}
k \\
j-1
\end{array}\right)
\end{aligned}
$$




$$
\begin{aligned}
& =1+2(k+1)+\sum_{j=2}^{\min \{s+1, k-i\}} 2^{j}\left(\begin{array}{c}
s+1 \\
j
\end{array}\right)\left(\begin{array}{c}
k \\
j-1
\end{array}\right) \\
& =1+2(k+1)-2(s+1)+\sum_{j=0}^{\min \{s+1, k-i\}} 2^{j}\left(\begin{array}{c}
s+1 \\
j
\end{array}\right)\left(\begin{array}{c}
k \\
j-1
\end{array}\right) \\
& =1+2(k-s)+\sum_{j=0}^{\min \{s+1, k-i\}} 2^{j}\left(\begin{array}{c}
s+1 \\
j
\end{array}\right)\left(\begin{array}{c}
k \\
j-1
\end{array}\right) .
\end{aligned}
$$

We are now in a position to prove our main result.

Proof of Theorem 1.6 For all sufficiently small $0<\delta \ll \varepsilon$ we have by Lemma 3.3 that the homology groups of $S_{\varepsilon}^{h}$ and $T_{\varepsilon, \delta}$ are isomorphic. Moreover, for $0 \leq i \leq k-$ $1, b_{i}(S)=\frac{1}{2} b_{i}\left(S_{\varepsilon}^{h}\right)$ by Proposition 3.1 and Lemma 3.2. Hence, the first inequality follows from Proposition 3.4.

The second inequality follows from an easy computation.

\section{Conclusion and Open Problems}

In this paper we have improved the upper bound proved by Barvinok on the Betti numbers of semi-algebraic sets in $\mathbb{R}^{k}$ defined by $s \leq \frac{k}{2}$ quadratic inequalities. The new bound is of the form $(O(k / s))^{s}$ improving the previous bound of $k^{O(s)}$ due to Barvinok. Using the fact that a complex non-singular complete intersection in $\mathbb{C}^{k}$ defined by $s$ quadratic equations can be viewed as a real semi-algebraic set in $\mathbb{R}^{2 k}$ defined $2 s$ quadratic equations, it follows that the best bound on the sum of the Betti numbers of semi-algebraic sets defined by $s$ quadratic inequalities in $\mathbb{R}^{k}$ cannot be better than $k^{\Omega(s)}$. However, this still does not prove that our bound is asymptotically tight. An interesting problem is to construct examples of sets, $S \subset \mathbb{R}^{k}$ defined by $s$ quadratic inequalities, such that $b(S) \geq(\Omega(k / s))^{s}$.

Another interesting problem in this context is to obtain a tighter bound on the number of connected components (that is on $b_{0}(S)$ ) for $S \subset \mathbb{R}^{k}$ defined by $s \leq k$ quadratic inequalities. It can be easily seen from the example of $S \subset \mathbb{R}^{k}$ defined by,

$$
X_{1}\left(X_{1}-1\right) \geq 0, \ldots, X_{s}\left(X_{s}-1\right) \geq 0 \text {, }
$$

that $b_{0}(S)$ can be as large as $2^{s}$. However, we know of no examples where $b_{0}(S)$ is as large as $k^{\Omega(s)}$. Note that the Betti numbers of a non-singular complex complete intersection is concentrated in the "middle" dimension. Consequently, the Smith inequality gives bounds only on the sum of the Betti numbers of the corresponding real varieties. Because of this reason, the method used in this paper cannot be used to prove better bounds on an individual (say the lowest or the highest) Betti number.

Acknowledgements The authors would like to thank Igor Belegradek for useful discussions, and Michel Coste for simplifying the proof of Proposition 2.3. 


\section{References}

1. Agrachev, A.A.: Topology of quadratic maps and Hessians of smooth maps. In: Algebra, Topology, Geometry, vol. 26, pp. 85-124, 162 (in Russian). Itogi Nauki i Tekhniki, Akad. Nauk SSSR, Vsesoyuz. Inst. Nauchn. Tekhn. Inform., Moscow, 1988. Translated in J. Sov. Math. 49(3), 990-1013 (1990).

2. Barvinok, A.I.: On the Betti numbers of semi-algebraic sets defined by few quadratic inequalities. Math. Z. 225, 231-244 (1997)

3. Barvinok, A.I.: Feasibility testing for systems of real quadratic equations. Discrete Comput. Geom. 10, 1-13 (1993)

4. Basu, S.: On bounding the Betti numbers and computing the Euler characteristic of semi-algebraic set. Discrete Comput. Geom. 22, 1-18 (1999)

5. Basu, S.: Different bounds on the different Betti numbers of semi-algebraic sets. Discrete Comput. Geom. 30, 65-85 (2003)

6. Basu, S.: Efficient algorithm for computing the Euler-Poincaré characteristic of semi-algebraic sets defined by few quadratic inequalities. Comput. Complex. 15, 236-251 (2006)

7. Basu, S.: Computing the top few Betti numbers of semi-algebraic sets defined by quadratic inequalities in polynomial time. Found. Comput. Math. (2007, in press)

8. Basu, S., Pollack, R., Roy, M.-F.: Algorithms in Real Algebraic Geometry, 2nd edn. Algorithms and Computation in Mathematics, vol. 10. Springer, Berlin (2006)

9. Basu, S., Pollack, R., Roy, M.-F.: On the Betti numbers of sign conditions. Proc. Am. Math. Soc. 133, 965-974 (2005)

10. Basu, S., Zell, T.: Polynomial time algorithm for computing certain Betti numbers of projections of semi-algebraic sets defined by few quadratic inequalities. Discrete Comput. Geom. (2007, to appear)

11. Benedetti, R., Loeser, F., Risler, J.J.: Bounding the number of connected components of a real algebraic set. Discrete Comput. Geom. 6, 191-209 (1991)

12. Gabrielov, A., Vorobjov, N.: Betti numbers of semialgebraic sets defined by quantifier-free formulae. Discrete Comput. Geom. 33, 395-401 (2005)

13. Grigor'ev, D., Pasechnik, D.V.: Polynomial time computing over quadratic maps I. Sampling in real algebraic sets. Comput. Complex. 14, 20-52 (2005)

14. Harris, J.: Algebraic Geometry: A First Course. Springer, Berlin (1992)

15. Hatcher, A.: Algebraic Topology. Cambridge University Press, Cambridge (2002)

16. Lewis, J.: A Survey of the Hodge Conjecture 2nd edn. CRM Monograph Series. American Mathematical Society, Providence (1999)

17. Massey, W.S.: A Basic Course in Algebraic Topology. Graduate Texts in Mathematics, vol. 127. Springer, Berlin (1991)

18. Milnor, J.: On the Betti numbers of real varieties. Proc. Am. Math. Soc. 15, 275-280 (1964)

19. Oleinik, O.A., Petrovskii, I.B.: On the topology of real algebraic surfaces. Izv. Akad. Nauk SSSR 13, 389-402 (1949)

20. Thom, R.: Sur l'homologie des varietes algebriques reelles. In: Cairns, S.S. (ed.) Differential and Combinatorial Topology, pp. 255-265. Princeton University Press, Princeton (1965)

21. Viro, O.Y., Fuchs, D.B.: In: Novikov, S.P., Rokhlin, V.A. (eds.) Topology II: Homology and Cohomology. Encyclopaedia of Mathematical Sciences, vol. 24. Springer, Berlin (2004) 